\title{
Manifestation of a spin-splitting field in a thermally biased Josephson junction
}

\author{
F. S. Bergeret ${ }^{1,2, *}$ and F. Giazotto ${ }^{3, \dagger}$ \\ ${ }^{1}$ Centro de Física de Materiales (CFM-MPC), Centro Mixto CSIC-UPV/EHU, Manuel de Lardizabal 4, E-20018 San Sebastián, Spain \\ ${ }^{2}$ Donostia International Physics Center (DIPC), Manuel de Lardizabal 5, E-20018 San Sebastián, Spain \\ ${ }^{3}$ NEST, Instituto Nanoscienze-CNR and Scuola Normale Superiore, I-56127 Pisa, Italy \\ (Received 6 December 2013; revised manuscript received 28 January 2014; published 11 February 2014)
}

\begin{abstract}
We investigate the behavior of a Josephson junction consisting of a ferromagnetic insulator-superconductor (FI-S) bilayer tunnel coupled to a superconducting electrode. We show that the Josephson coupling in the structure is strengthened by the presence of the spin-splitting field induced in the FI-S bilayer. Such strengthening manifests itself as an increase of the critical current $I_{c}$ with the amplitude of the exchange field. Furthermore, the effect can be strongly enhanced if the junction is taken out of equilibrium by a temperature bias. We propose a realistic setup to assess experimentally the magnitude of the induced exchange field, and predict a drastic deviation of the $I_{c}(T)$ curve ( $T$ is the temperature) with respect to equilibrium.
\end{abstract}

DOI: 10.1103/PhysRevB.89.054505

PACS number(s): 74.50.+r, 74.25.F-

The interplay between superconductivity and ferromagnetism in superconductor-ferromagnet (S-F) hybrids exhibits a large variety of effects studied along the last years [1,2]. Experimental research mainly focuses on the control of the $0-\pi$ transition in the S-F-S junctions $[3,4]$ (S-F-S) and on the creation, detection, and manipulation of triplet correlations in S-F hybrids [5-9]. From a fundamental point of view, the key phenomenon for the understanding of these effects is the proximity effect in S-F hybrids, and how the interplay between superconducting and magnetic correlations affect their thermodynamic and transport properties.

While most of the theoretical and experimental investigations on S-F structures deal mainly with the penetration of the superconducting order into the ferromagnetic regions, it is also widely known that magnetic correlations can be induced in the superconductor via the inverse proximity effect [10-13]. If the ferromagnet is an insulator (FI), on the one hand, superconducting correlations are weakly suppressed at the FI-S interface and a finite exchange field, with an amplitude smaller than the superconducting gap $\Delta_{0}$, is induced at the interface. Such exchange correlations penetrate into the bulk of $S$ over distances of the order of the coherence length [10]. This results in a splitting of the density of states (DOS) of the superconductor, as observed in a number of experiments [14-17]. Yet, the spin-split DOS of a superconductor may lead to interesting effects such as, for instance, the absolute spinvalve effect [18-20], the magnetothermal Josephson valve $[21,22]$, and the large enhancement of the Josephson coupling observed in F-S-I-S-F junctions (I stands for a conventional insulator) when the magnetic configuration of the $F$ layers is arranged in the antiparallel state [23-26].

In this paper, we show that an enhancement of the Josephson effect between two tunnel-coupled superconductors $S_{L}$ and $\mathrm{S}_{R}$ can also be achieved if a unique FI is attached to one of the S electrodes, for instance, the left lead, as shown schematically in Fig. 1(a). According to the discussion above, the presence of the FI splits the DOS in the left superconductor. In principle, the presence of the spin-splitting field causes

\footnotetext{
*sebastian_bergeret@ehu.es

† giazotto@sns.it
}

a reduction of the superconducting gap $\left(\Delta_{L}\right)$ in the left superconductor, and therefore at first glance one may think that, in turn, the Josephson coupling is suppressed. However, we show that for low enough temperatures, the presence of the induced exchange field $h$ in one of the two electrodes indeed enhances the critical current $\left(I_{c}\right)$ with respect to its value at $h=0$. This effect is further enhanced by applying a temperature bias across the junction. Furthermore, by setting $\mathrm{S}_{L}$ at $T_{L}$ and $\mathrm{S}_{R}$ at $T_{R}$, the temperature-dependent $I_{c}$ curves change drastically, showing a sharp step when the energy gap's difference matches the exchange field. A measurement of $I_{c}$ therefore allows us to assess the magnitude of the induced $h$. We discuss different realizations, and propose a realistic setup and materials combinations to demonstrate our predictions.

In order to understand the enhancement of the Josephson coupling by increasing the exchange field $h$, we provide here a simple physical picture that involves two mechanisms: On the one hand, the Josephson effect in the FI-S-I-S junction of Fig. 1(a) is stronger the larger the overlap of the condensates from the left and right electrodes is. This overlap can be thought of as being proportional to the number of Cooper pairs with shared electrons between $S_{L}$ and $S_{R}$. By increasing the exchange field in the left side of the junction, it is energetically more favorable for those pairs originally in $S_{L}$ to have the electrons with spin parallel to the field (spin-up) localized in the $S_{L}$ electrode, whereas spin-down electrons are preferably localized in $\mathrm{S}_{R}$ where the exchange field is absent. This means that the number of Cooper pairs sharing becomes larger. On the other hand, the Josephson coupling is proportional to the amplitude of the condensate in each of the electrodes. Therefore, by increasing $h$ or the temperature $(T)$, one expects a suppression of the order parameter in the electrodes or, equivalently, a suppression of the total number of pairs. The behavior of the Josephson critical current as a function of $h$ and $T$ is therefore the result of these two competing mechanisms. In the structure under consideration, the exchange field acts only in $\mathrm{S}_{L}$. For low enough temperatures, $\Delta_{L}(h, T)$ depends only weakly on $h$. Therefore, the first mechanism dominates and $I_{c}$ is enhanced by increasing $h$ [see Fig. 2(a)]. At large enough temperatures, $\Delta_{L}(h, T)$ is much more sensitive to the exchange field, and its faster suppression leads to a decrease 
(a)

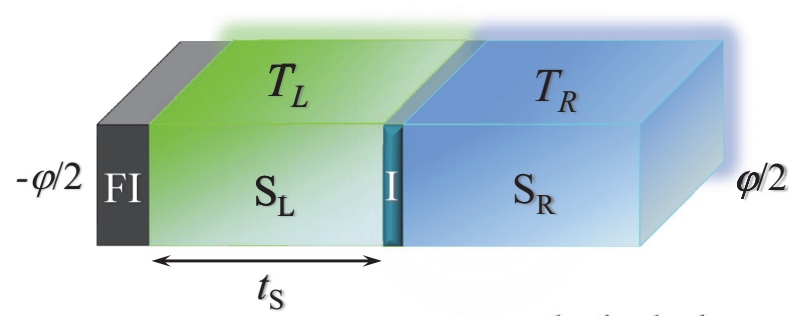

(b)

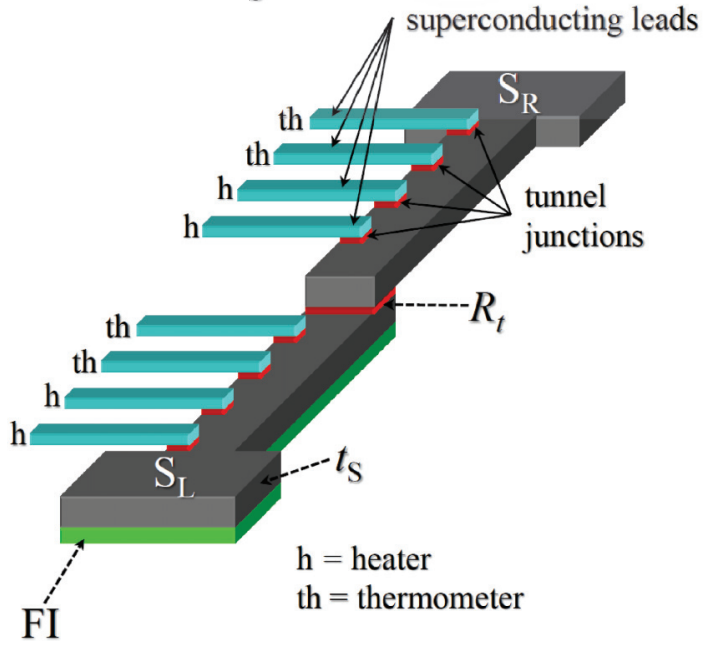

FIG. 1. (Color online) (a) Scheme of the FI-S-I-S Josephson tunnel junction considered in this paper. $T_{L}$ and $T_{R}$ indicate the temperature in the left $\left(\mathrm{S}_{L}\right)$ and right $\left(\mathrm{S}_{R}\right)$ superconductors, respectively, I stands for a conventional insulator, whereas $\varphi$ is the macroscopic quantum phase difference over the junction. $t_{\mathrm{S}}$ denotes the thickness of the $S_{L}$ layer. (b) Sketch of a possible experimental setup. Additional superconducting leads tunnel coupled to $S_{L}$ and $S_{R}$ serve either as heaters (h) or thermometers (th), and allow one to probe the effect of a spin-splitting field through measurement of the junction current vs voltage characteristics under conditions of a temperature bias, as discussed in the text. $R_{t}$ denotes the junction normal-state resistance.

of $I_{c}$ upon increasing $h$. We note that in $\mathrm{S}_{R}$ the exchange field is absent, and therefore the suppression of $\Delta_{R}$ is caused only by the increase of the temperature. If we now keep $S_{L}$ at low temperature and vary only the right electrode temperature $\left(T_{R}\right)$, the first mechanism dominates for any value of $T_{R}$, and the enhancement of $I_{c}$ by increasing $h$ can always be observed [cf. Fig. 2(b)]. This is a remarkable effect that we now analyze quantitatively in the following.

In order to compute the Josephson current through the junction sketched in Fig. 1(a), we assume that the normal-state resistance of the tunneling barrier $R_{t}$ is much larger than the normal-state resistances of the junction electrodes. In such a case, the charge current through the junction can be calculated from the well-known expression

$$
I=\frac{1}{32 e R_{t}} \int \operatorname{Tr}\left\{\tau_{3}\left[\mathbf{G}_{L}(E), \mathbf{G}_{R}(E)\right]^{K}\right\} d E,
$$

where the matrix $\tau_{3}$ in Eq. (1) is the third Pauli matrix in the particle/hole space, the Green's functions $(\mathrm{GFs}) \mathbf{G}_{L(R)}$ are the bulk GFs matrices for the left and right electrodes, and $e$ is the electron charge. They are $8 \times 8$ matrices in the

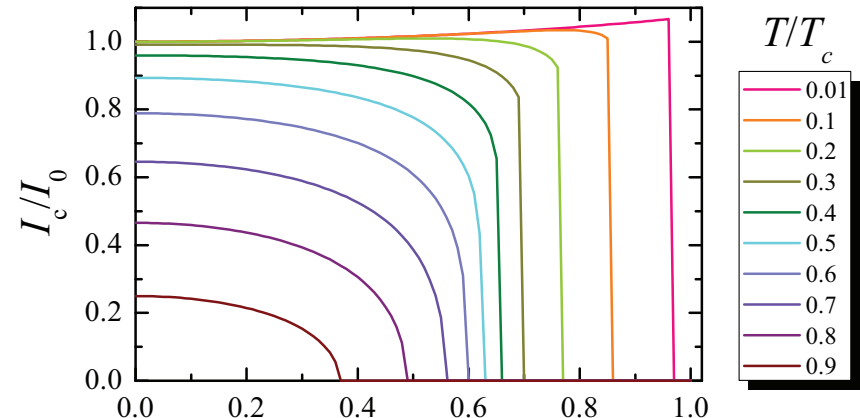

(a)

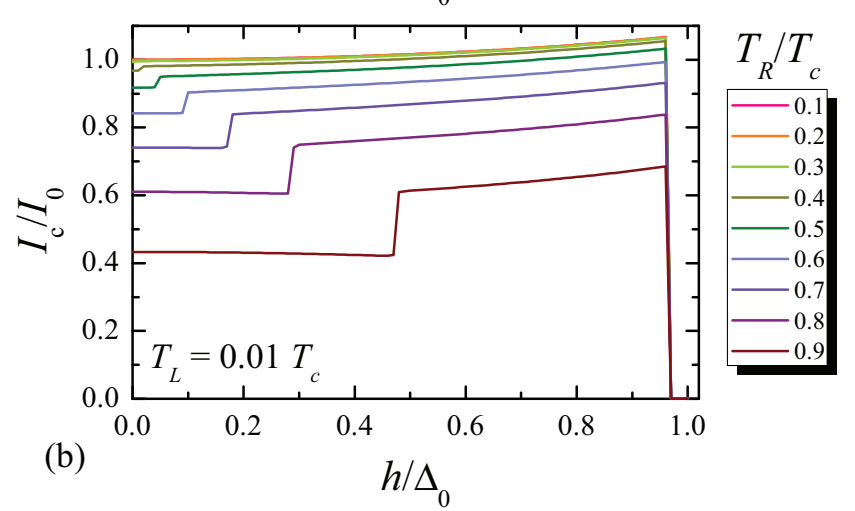

FIG. 2. (Color online) (a) Junction critical current $I_{c}$ versus exchange field $h$ calculated for several values of the temperature. Here, we set $T_{L}=T_{R}=T$. (b) Critical current $I_{c}$ versus $h$ calculated for different values of $T_{R}$ at $T_{L}=0.01 T_{c}$. $\Delta_{0}$ denotes the zero-field, zerotemperature superconducting energy gap with critical temperature $T_{c}$.

Keldysh-particle/hole-spin space with the structure

$$
\mathbf{G}_{L(R)}=\left(\begin{array}{cc}
\check{G}_{L(R)}^{R} & \check{G}_{L(R)}^{K} \\
0 & \check{G}_{L(R)}^{A}
\end{array}\right) .
$$

We assume that the junction is temperature biased so that the left (right) electrode is held at a constant and uniform temperature $T_{L(R)}$, and $\varphi$ denotes the macroscopic phase difference between the superconducting electrodes. In this case, the retarded (R) and advanced (A) components are $4 \times 4$ matrices in particle/hole-spin space defined as

$\breve{G}_{L(R)}^{R(A)}=\hat{g}_{L(R)}^{R(A)} \tau_{3}+\hat{f}_{L(R)}^{R(A)}\left[i \tau_{2} \cos (\varphi / 2) \pm i \tau_{1} \sin (\varphi / 2)\right]$,

where $\hat{g}$ and $\hat{f}$ are matrices in spin space defined by $\hat{g}_{L(R)}^{R}=$ $g_{-L(R)}^{R} \sigma_{3}+g_{+L(R)}^{R} \sigma_{0}$ and $\hat{f}_{L(R)}^{R}=f_{-L(R)}^{R} \sigma_{3}+f_{+L(R)}^{R} \sigma_{0}$. In principle, the functions in the left electrode may depend on the spatial coordinates. To simplify the problem, we assume that the thickness $t_{S}$ of the $\mathrm{S}_{L}$ electrode is smaller than the superconducting coherence length and hence the Green's functions are well approximated by spatially constant functions [27]

$$
\begin{aligned}
\hat{f}_{ \pm L(R)}^{R}= & \frac{1}{2}\left[\frac{\Delta_{L(R)}}{\sqrt{(E+h+i \Gamma)^{2}-\Delta_{L(R)}^{2}}}\right. \\
& \left. \pm \frac{\Delta_{L(R)}}{\sqrt{(E-h+i \Gamma)^{2}-\Delta_{L(R)}^{2}}}\right] .
\end{aligned}
$$


$\hat{g}_{ \pm L(R)}^{R}$ has a similar form by replacing $\Delta_{L(R)}$ in the numerators of the previous expressions with $E \pm h$. For the particular setup of Fig. 1(a), the exchange field in the right electrode is set to zero $(h=0)$ and therefore $g_{-L}=f_{-L}=0$ and $f_{R}=f_{+R}$. Notice that the gaps $\Delta_{L(R)}$ depend on the corresponding temperature $T_{L(R)}$ and exchange field, and have to be determined self-consistently. The advanced GFs have the same form after replacing $i \Gamma \rightarrow-i \Gamma$. The latter parameter describes inelastic effects within the time-relaxation approximation [33]. Finally, the Keldysh component of the GF [Eq. (2)] is defined as

$$
\check{G}_{L(R)}^{K}=\left(\check{G}_{L(R)}^{R}-\check{G}_{L(R)}^{A}\right) \tanh \left(E / 2 T_{L(R)}\right) .
$$

By using Eqs. (2)-(5), we can compute the electric current from Eq. (1). In the absence of a voltage drop across the junction (i.e., $V=0$ ), the charge current equals the Josephson current $I_{J}=I_{c} \sin \varphi$, where the critical supercurrent is given by the expression

$$
\begin{aligned}
I_{c}= & \frac{i}{8 e R_{t}} \int d E\left\{\left[f_{R}^{R} f_{+L}^{R}-f_{R}^{A} f_{+L}^{A}\right]\right. \\
& \times\left[\tanh \left(\frac{E}{2 T_{R}}\right)+\tanh \left(\frac{E}{2 T_{L}}\right)\right]+\left[f_{R}^{R} f_{+L}^{A}-f_{R}^{A} f_{+L}^{R}\right] \\
& \left.\times\left[\tanh \left(\frac{E}{2 T_{R}}\right)-\tanh \left(\frac{E}{2 T_{L}}\right)\right]\right\} .
\end{aligned}
$$

The second line of the above expression corresponds to the contribution from out-of-equilibrium conditions due to a temperature bias across the junction. It vanishes when both electrodes are held at the same temperature and, as we will see in the following, leads to important deviations of $I_{c}(T)$ from its equilibrium behavior.

Before analyzing the most general case, we first assume equilibrium, i.e., $T_{L}=T_{R}=T$ and compute the Josephson critical current as a function of the exchange field. This is shown in Fig. 2(a). At low enough temperatures, $I_{c}$ increases by increasing the exchange field. This is an unexpected result since the increase of the exchange field in the left electrode reduces the corresponding self-consistent gap $\Delta_{L}$, and therefore at first glance this suppression might lead to a reduction of $I_{c}$. However, this mechanism competes with the Josephson coupling, which, within the simple physical picture given in the introduction, is enhanced thanks to the fact that the electrons of the Cooper pairs with spin projection parallel to the field $h$ prefer to be localized mainly in $S_{L}$ while those with antiparallel spin are mostly localized in $\mathrm{S}_{R}$.

To quantify the effect, it is convenient to consider the limiting case $T \rightarrow 0$ so that the critical current [Eq. (6)] can be written as

$$
I_{c}^{e q}(T=0)=\frac{\Delta_{0}^{2}}{2 e R_{t}} \int \frac{d E}{\sqrt{E^{2}+\Delta_{0}^{2}}} \operatorname{Re}\left[\frac{1}{\sqrt{(E+i h)^{2}+\Delta_{0}^{2}}}\right]
$$

where $\Delta_{0}$ is the superconducting gap at $T=0$ and $h=0$. For small values of $h \ll \Delta_{0}$, one can expand this expression and find

$$
I_{c}^{e q}(T=0) \approx \frac{\pi \Delta_{0}}{2 e R_{t}}\left(1+\frac{1}{8} \frac{h^{2}}{\Delta_{0}^{2}}\right)
$$

which confirms the enhancement of $I_{c}$ upon increasing $h$. In the opposite limit, i.e., $h \rightarrow \Delta_{0}$, numerical evaluation of the integral gives $e R_{t} I_{c}^{e q}\left(T=0, h=\Delta_{0}\right) \approx 1.69 \Delta_{0}$ which is larger than the expected value at $h=0$, i.e., $\pi \Delta_{0} / 2$ [34]. This contrasts with what is obtained for the critical current of a F-S-I-S-F structure with magnetizations in the F layers arranged in the antiparallel configuration, which diverges as $h \rightarrow \Delta_{0}$ [23]. Therefore, although a larger effect can be achieved in a S-F-I-S-F (or FI-S-I-S-FI junction), for practical purposes the setup of Fig. 1(a) with just one single FI is much simpler, and the measurement of $I_{c}$ enhancement does not require control of the magnetization's direction. Moreover, in our geometry one can boost the supercurrent enhancement by applying a temperature bias across the junction, as we shall discuss in the following.

If the temperatures in the superconductors are different $\left(T_{L} \neq T_{R}\right)$, although each of the electrodes is in local steady-state equilibrium, the junction as a whole is in an out-ofequilibrium condition. In such a situation, also the second line in Eq. (6) contributes to the amplitude of the critical current, and leads to new features in the dependence of $I_{c}$ on $h$ and on the temperature difference. For instance, one can hold $S_{L}$ at some fixed $T_{L}$ and vary the temperature $T_{R}$ of $\mathrm{S}_{R}$, or vice versa. The critical current can be calculated numerically from Eq. (6). These results are shown in Fig. 2(b) where we set $T_{L}=0.01 T_{c}$, and $T_{R}$ varies from $0.1 T_{c}$ up to $0.9 T_{c}$. It is clear that, for large values of the spin-splitting field, $I_{c}$ is larger than the one for $h=0$. It is also remarkable that the effect is more pronounced the larger is the temperature difference. Furthermore, the main enhancement occurs stepwise, and stems from the out-of-equilibrium contribution to $I_{c}$ appearing in Eq. (6). The latter is equivalent to the expression for $I_{J_{1}}(V, T)$, the term proportional to $\sin \varphi$, of a voltage-biased Josephson junction obtained several years ago in Refs. [35,36]. In our system, the induced exchange field plays the role of the voltage bias and, in agreement with Refs. [35,36], the jump takes place at the value of $h$ for which the following condition is satisfied:

$$
\left|\Delta_{R}\left(T_{R}\right)-\Delta_{L}\left(T_{L}, h\right)\right|=h .
$$

We stress that while $I_{J_{1}}(V, T)$ can be accessed experimentally through a measurement of the ac Josepshon effect in voltage-biased configuration, the experiment we purpose below requires only a rather simple dc measurement at $V=0$.

In an experimental situation, it is somewhat difficult to tune in situ the exchange field present in the FI-S layer, and to verify the $I_{c}(h)$ dependence as displayed in Fig. 2. However, there is a simpler alternative way to proceed and to demonstrate these effects. Toward this end, we propose a possible experimental setup sketched in Fig. 1(b). The structure can be realized through standard lithographic techniques, and consists of a generic FI-S-I-S Josephson junction where the two $S_{L}$ and $\mathrm{S}_{R}$ electrodes are connected to additional superconducting (e.g., made of aluminum) probes through oxide barriers so to realize normal metal-insulator-superconductor (NIS) tunnel junctions. The NIS junctions are used to heat selectively the $\mathrm{S}_{L}$ or $\mathrm{S}_{R}$ electrode as well as to perform accurate electron thermometry [37]. Therefore, instead of varying the exchange field in the Josephson weak link, one could now hold one of the junction electrodes at a fixed temperature and vary the temperature of the other lead while recording the current versus 

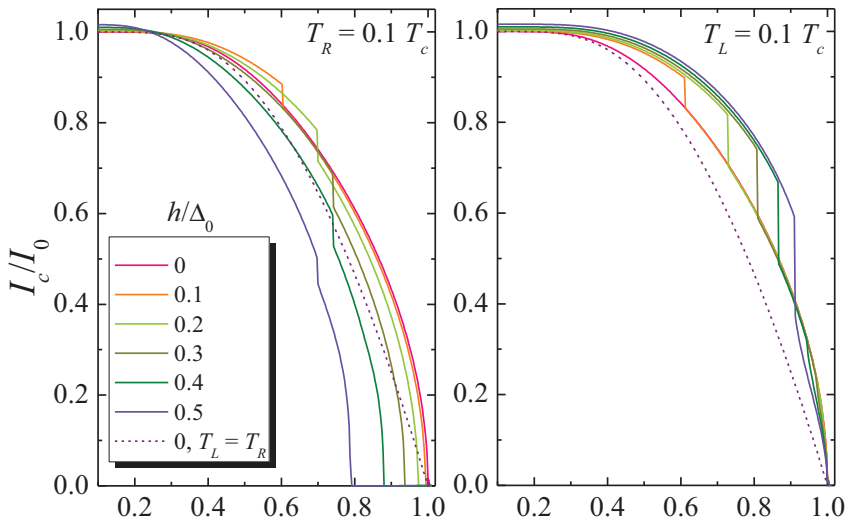

(a)

(b)

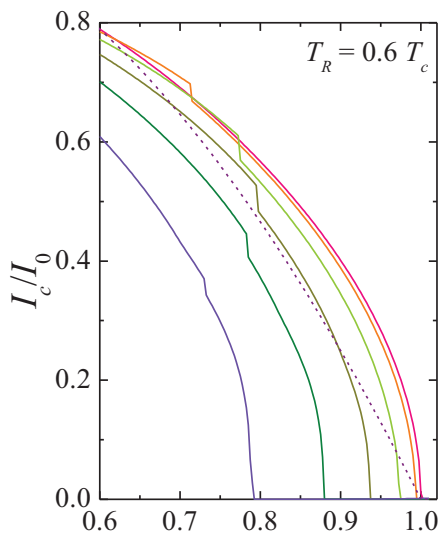

(c)

$$
T_{L} / T_{c}
$$

(d)

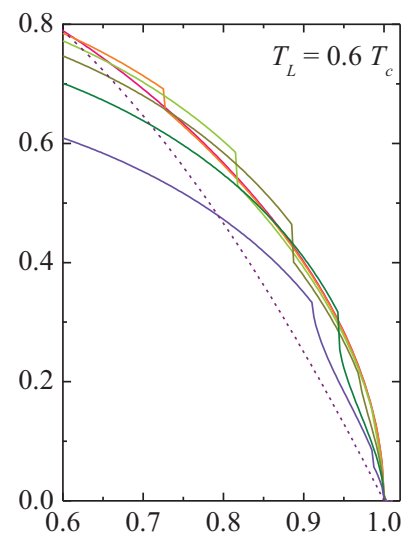

$$
T_{R} / T_{c}
$$

FIG. 3. (Color online) (a) Critical current $I_{c}$ versus $T_{L}$ calculated for selected values of the exchange field $h$ at $T_{R}=0.1 T_{c}$. (b) $I_{c}$ versus $T_{R}$ calculated at $T_{L}=0.1 T_{c}$ for the same values of $h$ as in panel (a). (c) The same as in panel (a) calculated for $T_{R}=0.6 T_{c}$. (d) The same as in panel (b) calculated for $T_{L}=0.6 T_{c}$. In all panels, the dashed line shows the critical current for $T_{L}=T_{R}$ and in the absence of an exchange field.

voltage characteristics under conditions of a temperature bias [38-42]. In this context, the electric current can be led through the whole structure via suitable outer superconducting electrodes allowing good electric contact, but providing the required thermal insulation necessary for thermally biasing the Josephson junction. In addition, the tunnel probes enable us to determine independently the energy gaps in the two superconducting electrodes through differential conductance measurements. Moreover, from the materials side, ferromagnetic insulators such as $\mathrm{EuO}$ or EuS $[14,15,43]$ combined with superconducting aluminum could be suitable candidates in light of a realistic implementation of the structure.

The critical current behavior under thermal-bias conditions is displayed in Fig. 3 where, in Figs. 3(a) and 3(c), $T_{R}$ is held at $0.1 T_{c}$ and $0.6 T_{c}$, respectively, and $T_{L}$ varies. Similarly, in Figs. 3(b) and 3(d), we keep $T_{L}$ constant at $0.1 T_{c}$ and vary $T_{R}$. It clearly appears that the $I_{c}(T)$ curves drastically deviate form those obtained at equilibrium, i.e., for $T_{L}=T_{R}$ (dotted lines in Fig. 3). If we keep a constant temperature $0.1 T_{c}$ in one of the electrodes [see Figs. 3(a) and 3(b)] and change the temperature of the other, it follows that, for low enough temperatures, $I_{c}$ gets larger by increasing the magnitude of the exchange field. This corresponds to the enhancement discussed in Fig. 2. Further increasing the temperature of one of the electrodes leads to a critical current decrease. Notably, $I_{c}$ exhibits a sharp jump at those temperatures such that the condition expressed by Eq. (9) holds. This is a striking effect which can provide, from the experimental side, evidence of the supercurrent enhancement discussed above. Yet, it can be used as well to determine the value of the effective exchange field induced in the superconductor placed in direct contact with the FI layer. It is remarkable that these features can be also observed, although reduced in amplitude, in the high-temperature regime [see Figs. 3(c) and 3(d)]. We emphasize that the effect here discussed is much more pronounced when the left electrode (i.e., the one with the FI layer) is kept at a low temperature, and one varies $T_{R}$. This is simple to understand since a superconductor with a spin-splitting field is more sensitive to a temperature variation: the larger the exchange field, the faster one gets suppression of superconductivity by enhancing the temperature.

In conclusion, we have shown that the critical current $I_{c}$ of a FI-S-I-S Josephson junction is drastically modified by the presence of the exchange field induced in one of the electrodes from the contact with a ferromagnetic insulator. In particular, we have demonstrated that the Josephson coupling is strengthened by the presence of the exchange field and therefore the $I_{c}$ amplitude is enhanced. The enhancement becomes more pronounced upon the application of a temperature bias across the junction. In such a case, we predict a change of the $I_{c}(T)$ curve with respect to the equilibrium situation which now shows a jump occurring when the difference of the superconducting gaps equals the amplitude of the exchange field. This behavior can be measured through standard techniques as we have discussed for a realistic experimental setup. Our predictions on Josephson junctions with ferromagnetic insulators are of great relevance since they constitute the building blocks of recently proposed nanodevices for spintronics [19,44] and coherent spin caloritronics [21,22].

The work of F.S.B was supported by the Spanish Ministry of Economy and Competitiveness under Project No. FIS201128851-C02-02. F.G. acknowledges the Italian Ministry of Defense through the PNRM project "Terasuper", and the Marie Curie Initial Training Action (ITN) Q-NET 264034 for partial financial support. F.S.B thanks M. Holthaus and his group for their kind hospitality at the Physics Institute of the Oldenburg University.
[1] F. S. Bergeret, A. F. Volkov, and K. B. Efetov, Rev. Mod. Phys. 77, 1321 (2005).

[2] A. I. Buzdin, Rev. Mod. Phys. 77, 935 (2005).

[3] A. I. Buzdin, L. N. Bulaevskii, and S. V. Panyukov, Pis'ma Zh. Eksp. Teor. Fiz. 35, 147 (1982) [JETP Lett. 35, 178 (1982)].
[4] V. V. Ryazanov, V. A. Oboznov, A. Yu. Rusanov, A. V. Veretennikov, A. A. Golubov, and J. Aarts, Phys. Rev. Lett. 86, 2427 (2001).

[5] F. S. Bergeret, A. F. Volkov, and K. B. Efetov, Phys. Rev. Lett. 86, 4096 (2001). 
[6] R. S. Keizer, S. T. B. Goennenwein, T. M. Klapwijk, G. Miao, G. Xiao, and A. Gupta, Nature (London) 439, 825 (2006).

[7] J. W. A. Robinson, J. D. S. Witt, and M. G. Blamire, Science 329, 59 (2010).

[8] T. S. Khaire, M. A. Khasawneh, W. P. Pratt, Jr., and N. O. Birge, Phys. Rev. Lett. 104, 137002 (2010); C. Klose, T. S. Khaire, Y. Wang, W. P. Pratt, Jr., Norman O. Birge, B. J. McMorran, T. P. Ginley, J. A. Borchers, B. J. Kirby, B. B. Maranville, and J. Unguris, ibid. 108, 127002 (2012).

[9] M. S. Anwar, F. Czeschka, M. Hesselberth, M. Porcu, and J. Aarts, Phys. Rev. B 82, 100501 (2010).

[10] T. Tokuyasu, J. A. Sauls, and D. Rainer, Phys. Rev. B 38, 8823 (1988).

[11] F. S. Bergeret, A. F. Volkov, and K. B. Efetov, Phys. Rev. B 69, 174504 (2004).

[12] F. S. Bergeret, A. Levy Yeyati, and A. Martin-Rodero, Phys. Rev. B 72, 064524 (2005).

[13] J. Xia, V. Shelukhin, M. Karpovski, A. Kapitulnik, and A. Palevski, Phys. Rev. Lett. 102, 087004 (2009).

[14] X. Hao, J. S. Moodera, and R. Meservey, Phys. Rev. B 42, 8235 (1990).

[15] T. S. Santos, J. S. Moodera, K. V. Raman, E. Negusse, J. Holroyd, J. Dvorak, M. Liberati, Y. U. Idzerda, and E. Arenholz, Phys. Rev. Lett. 101, 147201 (2008).

[16] G. Catelani, X. S. Wu, and P. W. Adams, Phys. Rev. B 78, 104515 (2008).

[17] Y. M. Xiong, S. Stadler, P. W. Adams, and G. Catelani, Phys. Rev. Lett. 106, 247001 (2011).

[18] R. Meservey and P. M. Tedrow, Phys. Rep. 238, 173 (1994).

[19] D. Huertas-Hernando, Yu V. Nazarov, and W. Belzig, Phys. Rev. Lett. 88, 047003 (2002).

[20] F. Giazotto and F. Taddei, Phys. Rev. B 77, 132501 (2008).

[21] F. Giazotto and F. S. Bergeret, Appl. Phys. Lett. 102, 132603 (2013).

[22] F. S. Bergeret and F. Giazotto, Phys. Rev. B 88, 014515 (2013).

[23] F. S. Bergeret, A. F. Volkov, and K. B. Efetov, Phys. Rev. Lett. 86, 3140 (2001).

[24] V. N. Krivoruchko and E. A. Koshina, Phys. Rev. B 64, 172511 (2001).
[25] A. V. Zaitsev, JETP Lett. 90, 475 (2009).

[26] J. W. A. Robinson, G. B. Halász, A. I. Buzdin, and M. G. Blamire, Phys. Rev. Lett. 104, 207001 (2010).

[27] For a thin enough S layer in contact with a FI, the Green's functions (4) can be obtained by integrating the Usadel equation over the thickness of $\mathrm{S}$ and using the boundary conditions at the FI layer derived in Refs. [28, 29]. This procedure has been used in Ref. [30], where it has been shown the connection between the induced exchange field and the FI/S interface parameters [31, 32].

[28] A. Cottet, D. Huertas-Hernando, W. Belzig, and Y. V. Nazarov, Phys. Rev. B 80, 184511 (2009).

[29] F. S. Bergeret, A. Verso, and A. F. Volkov, Phys. Rev. B 86, 214516 (2012).

[30] A. Cottet, Phys. Rev. Lett. 107, 177001 (2011).

[31] A. Cottet, Phys. Rev. B 76, 224505 (2007).

[32] A. Cottet and J. Linder, Phys. Rev. B 79, 054518 (2009).

[33] Throughout the paper, we set $\Gamma=10^{-6} \Delta_{0}$ for the numerical evaluation of $I_{c}$.

[34] M. Tinkham, Introduction to Superconductivity, 2nd ed. (McGraw-Hill, New York, 1996).

[35] A. I. Larkin and Yu. N. Ovchinnikov, J. Exptl. Theoret. Phys. (U.S.S.R.) 51, 1535 (1966) [Sov. Phys.-JETP 24, 1035 (1967)].

[36] R. E. Harris, Phys. Rev. B 10, 84 (1974).

[37] F. Giazotto, T. T. Heikkilä, A. Luukanen, A. M. Savin, and J. P. Pekola, Rev. Mod. Phys. 78, 217 (2006).

[38] S. Tirelli, A. M. Savin, C. P. Garcia, J. P. Pekola, F. Beltram, and F. Giazotto, Phys. Rev. Lett. 101, 077004 (2008).

[39] A. M. Savin, J. P. Pekola, J. T. Flyktman, A. Anthore, and F. Giazotto, Appl. Phys. Lett. 84, 4179 (2004).

[40] A. F. Morpurgo, T. M. Klapwijk, and B. J. van Wees, Appl. Phys. Lett. 72, 966 (1998).

[41] H. Courtois, M. Meschke, J. T. Peltonen, and J. P. Pekola, Phys. Rev. Lett. 101, 067002 (2008).

[42] S. Roddaro, A. Pescaglini, D. Ercolani, L. Sorba, F. Giazotto, and F. Beltram, Nano Res. 4, 259 (2011).

[43] G.-X. Miao, M. Müller, and J. S. Moodera, Phys. Rev. Lett. 102, 076601 (2009).

[44] F. Giazotto and F. S. Bergeret, Appl. Phys. Lett. 102, 162406 (2013). 